\title{
The Effects of Transcranial Direct Current Stimulation on Obsession-compulsion, Anxiety, and Depression of a crossuark Patient Suffering from Obsessive-compulsive Disorder
}

\author{
Jaber Alizadeh Goradel ${ }^{1}$, Asghar Pouresmali ${ }^{1}$, Mehri Mowlaie ${ }^{2 *}$, Fariba Sadeghi Movahed ${ }^{3}$ \\ 1. Young Researcher and Elite Club, Islamic Azad University, Ardabil Branch, Ardabil, Iran. \\ 2. Department of Psychology, Faculty of Education and Psychology, University of Mohaghegh Ardabili, Ardabil, Iran. \\ 3. Department of Psychiatry, School of Medicine \& Allied Medical Sciences, Ardabil University of Medical Science, Ardabil, Iran.
}

Crtation: Alizadeh Goradel, J., Pouresmali, A., Mowlaie, M., \& Sadeghi Movahed, F. (2016). The effects of transcranial direct current stimulation on obsession-compulsion, anxiety, and depression of a patient suffering from obsessive-compulsive disorder. Journal of Practice in Clinical Psychology, 4(2), 75-80. http://dx.doi.org/10.15412/J.JPCP.06040201

dof: $:$ http://dx.doi.org/10.15412/J.JPCP.06040201

\section{Article info:}

Received: 24 Nov. 2015

Accepted: 14 Feb. 2016

\section{Keywords:}

Anxiety, Depression, Transcranial direct current stimulation, Obsessioncompulsion

\begin{abstract}
A BSTRACT
Objective: Obsessive-Compulsive disorder (OCD) is one of the greatest and debilitating disorders, which many of its patients do not respond to psychotherapy and pharmacotherapy. In this regard, paying attention to new treatments such as transcranial direct current stimulation (tDCS) is important. The purpose of the present research was to examine the effects of tDCS on the obsession-compulsion (OC), anxiety, and depression of a patient suffering from OCD.

Methods: The present study conducted on a 23-year-old female patient with OCD. tDCS sessions were lasted 10 days, one session per day and each session of about 20 minutes with 2.0 $\mathrm{mA}$ intensity. The results of Beck depression inventory, Hamilton anxiety rating scale, and Yale Brown obsessive-compulsive scale showed decrease in OC, depression, and anxiety symptoms after 10 sessions of tDCS.

Results: The results of the present research using cathodal tDCS on orbitofrontal cortex in 10 sessions showed that this montage is a powerful and successful treatment for patients with OCD and alleviate their OC, depression, and anxiety symptoms.

Conclusion: The data were analyzed by diagram analysis and improvement percentage. This study showed that anodal tDCS on $\mathrm{O} 2$ and cathodal tDCS on orbitofrontal cortex improved OC, anxiety and depression symptoms. However, our findings give introductory confirmation that our patient with OCD has a positive response to cathode tDCS of the OFC.
\end{abstract}

\section{Introduction}

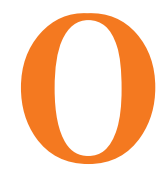

bsessive-compulsive disorder (OCD) is a debilitating psychiatric disorder with $2 \%$ lifetime prevalence (Ruscio, Sten, ldvin,
Chiu, \& Kessler, 2010). It is one of the most prevalent psychological disorders. OCD leads to terrible impairments in relationship with other people and occupational responsibility (Fontenelle, Fontenelle, \& Borges, 2010). It is supposed that OCD will become the sixth most debilitating psychi-

* Corresponding Author:

Mehri Mowlaie, PhD Student

Address: Department of Psychology, Faculty of Education and Psychology, University of Mohaghegh Ardabili, Ardabil, Iran.

Tel: +98 (914) 2908144

E-mail:mehri.molaee@yahoo.com 
atric disorder in 2015 (Mathers, et al., 2003). Only 40\% to $60 \%$ of these patients respond to available treatments such as pharmacotherapy (Foa, Liebowitz, \& Kozak, 2005), also cognitive-behavior therapy was effective only for some of these patients (Foa, Liebowitz, \& Kozak, 2005).

In many research studies, OCD is comorbid with depression and anxiety (Ivarsson, Melin, \& Wallin, 2008; Peris et al., 2010). Depression leads to drawback of treatment outcomes for individuals with OCD (Storch et al., 2008). Anxiety also is more prevalent than depression in OCD (Ivarsson, Melin, \& Wallin, 2008; Langley, Lewin, Bergman, Lee, \& Piacentini, 2010). The severity of anxiety will be great if the symptoms of OCD increase (Langley et al., 2010). Regarding the high prevalence of OCD and its demolishing effects on patients' life, it seems necessary to consider new technologies to treat OCD patients.

According to conducted research studies over past 20 years, there is an increased activity in the orbitofrontal cortex (OFC) in patients with OCD (Del Casale et al., 2011; Milad \& Rauch, 2012), including the dorsolateral prefrontal cortex (DLPFC), orbitofrontal cortex (OFC), medial prefrontal cortex, anterior cingulated gyrus, supplementary motor area (SMA), and basal ganglia (Shah, Pesiridou, \& Baltuch, 2008). The connections of the ventral striatum with prefrontal cortices are considered to be increased in these patients (Del Casale et al., 2011; Shah, Pesiridou, \& Baltuch, 2008). OFC plays a major role in the pathophysiology of OCD because obsessions and compulsions are mediated by hyper activity of the orbito-frontal cortex either bilaterally (Alptekin et al., 2001) or unilaterally (left side) (Baxter, Schwartz, \& Mazziotta, 1998; Swedo et al., 1992).

Further pathophysiological insight came from the application of the noninvasive brain stimulation techniques, i.e. transcranial magnetic stimulation (TMS) and transcranial direct current stimulation (tDCS). Noninvasive brain stimulation techniques such as tDCS is a vigorous and convenient technique to moderate brain activity. Anodal tDCS increases excitability of cortex in the special area of the brain, while cathodal tDCS decreases excitability of cortex in the specified area of the brain (Nitsche \& Paulus, 2000) by producing low-intensity electric field $(<1 \mathrm{~V} / \mathrm{m})$ (Datta et al., 2009).

In other words, cathodal and anodal stimulations hyperpolarize and depolarize neurons respectively (Priori, Hallet, \& Rothwell, 2009; Nitsche et al., 2003). This technique has shown optimistic results in many neuropsychiatric illnesses (Kuo, Paulus, \& Nitsche, 2014) and also has some advantages such as low cost, ease of use, portability, and safety (Volpato et al., 2013; Brunoni et al., 2011) over other techniques such as repetitive transcranial magnetic stimulation.
Previous studies have reported that noninvasive techniques such as tDCS alleviates the symptoms of treatment-resistant OCD (Jaafari et al., 2012). Volpato et al. (2013) found out that cerebellar-tDCS on DLPFC decreased anxiety and depressive symptoms but not OC symptoms in patients with treatment-resistant OCD. OC symptoms decreased when stimulation was delivered over the SMA or the OFC (Berlim, Neufeld, \& Van den Eynde, 2013). In a study by Mondino, Haesebaert, Poulet, Saoud and Brunelin (2015) which used cathodal tDCS on the left OFC (above FP1) and anodal tDCS on the contralateral orbitofrontal region (above $\mathrm{O} 2)$, they observed considerable decrease $(26 \%)$ in symptoms of patients with treatment-resistant OCD.

According to the mentioned studies, cathodal tDCS over the pre-SMA, (rather than anodal tDCS) significantly alleviates OCD symptoms (Senco et al., 2015), but it has not yet been identified whether tDCS can reduce simultaneously depression, anxiety, OC symptoms or not. In the present study, we used tDCS because it has an impact on corticostriatal and corticothalamic loops involved in the pathology of OCD (Polania, Paulus, \& Nitsche, 2012). Besides, we used Beck depression inventory (BDI) and Hamilton anxiety rating scale (HARS) tests to evaluate the impact of tDCS on depression, anxiety, and OC symptoms.

Considering that a few studies have directly dealt with this issue (no research in Iran and a few in abroad), as well as the novelty of tDCS, the present study is the first to investigate the effect of tDCS efficacy on the treatment for OCD, and especially to evaluate the effect of tDCS on depression, anxiety, and OC symptoms.

\section{Methods}

In this study, single-subject experimental trial was used in which, firstly, in stage A, primary condition of a patient was assessed by Yale Brown obsessive compulsive scale (YBOCS), Beck depression inventory (BDI), and Hamilton anxiety rating scale (HARS). Then, in stage B, experimental trial was done and the subject's condition in these scales was again assessed in follow up stage. Our participant was a 23-year-old female student, diagnosed with obsessivecompulsive disorder (based on DSM-V criteria) which was referred by a psychiatrist to receive noninvasive brain stimulation treatment because of the failure in response to other treatments. She had a one year history of this disease characterized by obsessions (religious type) without compulsion. Previous treatment with sertraline (for 3 months) did not improve her symptoms. Obsession symptoms of patients began one year ago when university exams started. Obsession symptoms were not found in her family in Minimental state examination. She had normal communication 
with her family and friends and did not report any problem in these areas. Patient was informed about the purpose of the study, and gave her written informed consent.

The cathode electrode was placed on the left orbito frontal cortex (OFC) and anode electrode on the contra lateral region $(\mathrm{O} 2)$. We used a direct current of $2.0 \mathrm{~mA}$ for 20 minutes per day for 10 sessions. The $25-\mathrm{cm}^{2}$ rubber electrodes with cotton material, which was moistened with saline to reduce impedance were used. For assessment of obsession/compulsion, anxiety, and depression symptoms, we used the YBOCS, HARS, and BDI at the first, fifth, and last session of the treatment and 2 weeks later as follow up. It is important to note that tDCS was started after providing consent form and this research took place at a clinical psychology clinic (a noninvasive center). Obsession, depression, and anxiety symptoms improved during the 10 session's of treatment.

The inclusion criteria included 1) Diagnosis of OCD by psychiatrist according to the DSMV and psychological interview, 2) Y-BOCS scores of 17 points or higher (Goodman et al., 1989), 3) Under no other therapy such as psychotherapy or pharmacotherapy, 4) Without comorbid psychiatric disorders, and 5) No drug abuse. The exclusion criteria included 1) Having metal implants, 2) Not meeting DSMV criteria for OCD, 3) Using drugs such as clomipramine, bupropion.

Yale-Brown obsessive compulsive scale (Y-BOCS): This scale was developed by Goodman et al., (1989) to measure OCD. It consists of 10 observer-rated items scored on a 5-point Likert-type scale. It is widely used in clinical trials and its administration only takes about 10 to 15 minutes. The scale has a good reliability and validity. Range of severity includes: $0-7=$ Subclinical, $8-15=$ Mild, $16-23=$ Moderate, 24-31=Severe, and 32-40=Extreme. In a study conducted by Rajezi Esfahani, Motaghipour, Kamkari, Zahiredin and Janbozorgi (2012), levels of internal consistency scores for symptom checklist was 0.97 and for severity scale 0.95 . Also, split-half reliability for symptom checklist was 0.93 and for severity scale 0.89 . Its test-retest reliability was 0.99 .

Hamilton anxiety rating scale (HARS):This scale was developed by Hamilton (1959). It is widely used as an as- sessment instrument for anxiety symptoms in psychiatry, and is designed to evaluate changes in symptoms over time. It consists of 14 items that cover 13 symptoms of anxiety. Each item is rated on a 4 -point scale $(0=$ not present to $4=$ severe) on an unanchored severity scale. The score range is $0-56$. Those who score 5 or less are considered as having no anxiety. Other categories are as follows: 6-14=mild anxiety; $15-28=$ moderate anxiety; $29-42=$ severe anxiety, and 43-56=very severe anxiety. In Iran, HARS has been validated by Kavyani, Mossavi and Mohit (2001) and was reported to have high reliability and validity.

Beck Depression Inventory (BDI): This scale is a 21-item, self-rating scale, which assesses depression. It consist of different symptoms of depression such as (i.e. agitation, worthlessness, concentration difficulty, loss of energy, weight loss, body image change, work difficulty, and somatic preoccupation), which rated from 0 to 3 with 4 possible answers. The Chronbach's alpha for BDI was $=0.86$ and for BDI-II was $=0.92$ which represent high reliabilty (Psychological Corporation Website, 2003). The standard cut-off scores are as follows: $0-9=$ minimal, $10-18=$ mild, $19-29=$ moder ate, $30-63=$ severe. Higher total scores indicate more severe depressive symptoms. Internal consistency of this scale in Iranian students was 0.87 , and its test-retest reliability in a week was 0.73 (Dobson \& Mohammad Khani, 2007).

\section{Results}

For analyzing the data and calculating changes, diagram analysis and improvement percentage were used. Table 1 shows the descriptive statistics of YBOCS, BDI, and HARS scores from first to last and follow up sessions. At the first session of the treatment, the patient scored 28 in YBOCS, 32 in HARS and 48 in BDI.

As seen in Table 1, in baseline, the scores of the patient regarding Y-BOCS was 28, in BDI was 48, and in HARS was 32. After 10 sessions of treatment, the scores of these scales reduced significantly, and after 2 weeks of treatment, the patient reported significantly suitable respones to this treatment (Figure 1). Also, there were no significant clinical or cognitive side effects. Improvement percentage for Y-BOCS was 64, for BDI was 87, and for HARS was 100.

Table 1. Descriptive statistics of the Y-BOCS, BDI, and HARS scores.

\begin{tabular}{|c|c|c|c|c|c|c|c|c|c|c|c|}
\hline \multicolumn{3}{|c|}{ Y-BOCS score } & \multicolumn{4}{|c|}{ BDI score } & \multicolumn{5}{|c|}{ HARS score } \\
\hline Baseline & $\begin{array}{l}\text { After } 5 \\
\text { days }\end{array}$ & $\begin{array}{c}\text { After } 10 \\
\text { days }\end{array}$ & Follow up & baseline & $\begin{array}{c}\text { After } 5 \\
\text { days }\end{array}$ & $\begin{array}{c}\text { After } 10 \\
\text { days }\end{array}$ & Follow up & Baseline & $\begin{array}{c}\text { After } 5 \\
\text { days }\end{array}$ & $\begin{array}{c}\text { After } 10 \\
\text { days }\end{array}$ & $\begin{array}{c}\text { Follow } \\
\text { up }\end{array}$ \\
\hline 28 & 13 & 10 & 8 & 48 & 21 & 6 & 5 & 32 & 19 & 0 & 0 \\
\hline
\end{tabular}



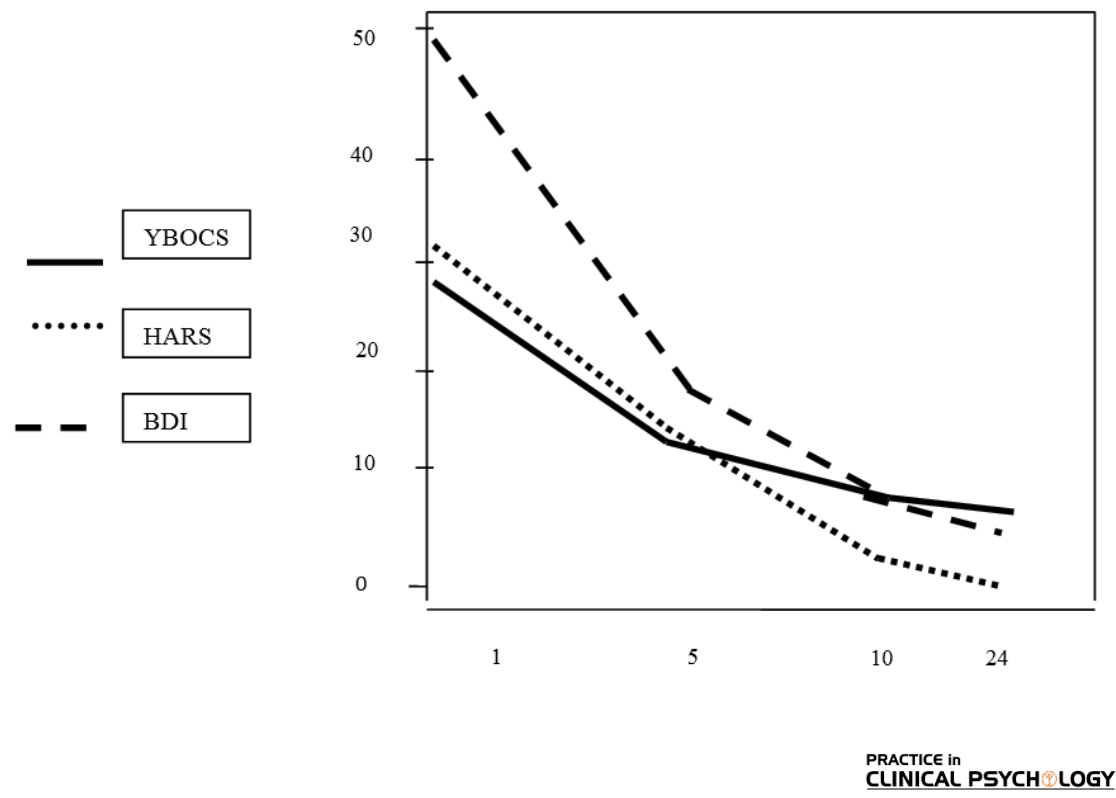

Figure 1. Y-BOCS, BDI, and HARS scores at baseline, the fifth day, last day, and $14^{\text {th }}$ days follow up.

These percentages indicated that $\mathrm{tDCS}$ has significant therapeutic effects on OC, depression, and anxiety.

\section{Discussion}

Noninvasive brain stimulation techniques have found progressive applications in the OCD field, providing an important contribution to our understanding of OCD pathophysiology, and offering new opportunities for treatment. The present study aimed to assess the decrease in obsession, depression, and anxiety symptoms via tDCS in a patient with OCD. Evidently, hyperactivation of prefrontal-thalamic circuits and the lack of inhibition of the cortico-striato-thalamo-cortical pathways are implicated in OCD pathophysiology (Alexander, Crutcher, \& DeLong, 1989).

According to this theory, DLPFC, OFC, ACC and striatum (specifically the caudate) will be the most important structures (Aronson, Katnani, \& Eskandar, 2014) involving in depression, anxiety, and obsession-compulsion. In line with previous studies, we found that cortical cathodal stimulation on OFC will decrease obsession (Jaffari et al., 2012; Berlim, Neufeld, \& Van den Eynde, 2013; Mondino et al., 2015) depression, and anxiety (Volpato et al., 2013). The present research is inconsistent with Volpato et al. (2013) which stated that tDCS only decrease anxiety and depressive symptoms but not OC symptoms. Our results showed that $\mathrm{tDCS}$ can have significant equal impact on depression, anxiety, and OC symptoms.
It is important to note that Mondino et al. (2015) in their research found after 10 sessions of tDCS, the symptoms of OC did not subside immediately after 10 sessions of tDCS but our patient showed considerable decrease in depression, anxiety, and OC symptoms after 10 sessions of tDCS. In fact, the symptoms of OC reduced $64.28 \%$, symptoms of anxiety $87.5 \%$, and depression $87.5 \%$ after 10 sessions. In order to confirm our results, the patient was asked to complete Y-BOCS, HARS, and BDI after 2 weeks, and the results showed decrease in these symptoms after this interval.

Our other results showed that tDCS decreases anxiety symptoms in OCD patient. This finding is consistent with some other studies (Del Casale et al., 2011; Polonia, Paulus, \& Nitsche, 2012; Sakai et al., 2011). Dysfunction in caudate nucleus leads to hyperactivity in orbitofrontal and anterior cingulate cortices which are respomsible areas of obsessive thought and anxiety. According to Del Casale et al., (2011) and Sakai et al. (2011), the contact of the ventral striatum with prefrontal cortices are considered to increased in these patients.

Plonia et al. (2012) showed that tDCS has an impact on functional cortico subcortical networks, including corticostriatal, and cortico-thalamic loops, which are involved in these disorders. The other results of our research showed that tDCS has incredible effect on depression. This finding is consistent with the findings of other studies (Fregni et al., 2006; Boggio et al., 2008; Loo et al., 2012; Bennabi et al., 2015). According to Berlim, Neufeld and Van den Eynde (2013), the rationale for considering tDCS as an effective 
treatment in depression is inducing functional changes in resting membrane potential and cerebral blood flow.

Like other studies, our research has some limitations too. First of all, it is a case study; therefore our results may not be generalized to other patients with OCD. To resolve this limitation, further controlled studies in large patient groups are needed. However, these results suggest that tDCS inhibit the orbitofrontal cortex while activate the occipital area above $\mathrm{O} 2$ has an interesting therapeutic potential in obsession-compulsion disorder. Therefore, in a practical way, this montage can be used by therapists to simultaneously treat obsession, anxiety, and depression symptoms.

In conclusion, the results of the present research showed that tDCS by using anode electrode on $\mathrm{O} 2$ and cathode electrode on OFC would safely, conveniently, and inexpensively decrease OC, anxiety, and depression symptoms in patients with OCD. Also as few studies has been done to investigate the impact of brain stimulation on these patients, this study might be the practical one and guide behavior therapists to combine their therapies with noninvasive brain stimulation in order to increase beneficial effects of tDCS.

\section{References}

Alexander, G. E., Crutcher, M. D., \& DeLong, M. R. (1989). Basal ganglia-thalamocortical circuits: parallel substrates for motor, oculomotor, "prefrontal" and "limbic" functions. Progress in Brain Research, 85, 119-146.

Alptekin, K., Degirmenci, B., Kivircik, B., Durak, H., Yemez, B., \& Derebek, E. (2001). Tc-99m HMPAO brain perfusion SPECT in drug-free obsessive-compulsive patients without depression. Psychiatry Research, 107(1), 51-56.

Aronson, J. P., Katnani, H. A., \& Eskandar, E. N. (2014). Neuromodulation for obsessive-compulsive disorder. Neurosurgs Clinical North America, 25(1), 85-101.

Baxter, L. R., Schwartz, J. M., \& Mazziotta, J. C. (1998). Cerebral glucose metabolic rates in non-depressed patients with obsessivecompulsive disorder. American Journal of Psychiatry, 145(12), 1560-1563.

Bennabi, D., Nicolier, M., Monnin, J., Tio, G., Pazart, L., Vandel, P., \& et al. (2015). Pilot study of feasibility of the effect of treatment with tDCS in patients suffering from treatment-resistant depression treated with escitalopram. Clinical Neurophysiology, 126(6), $1185-1189$

Berlim, M. T., Neufeld, N. H., \& Van den Eynde, F. (2013). Repetitive transcranial magnetic stimulation (rTMS) for obsessivecompulsive disorder (OCD): An exploratory meta-analysis of randomized and sham-controlled trials. Journal of Psychiatric Research, 47(8), 999-1006.
Boggio, P. S., Rigonatti, S. P., Ribeiro, R. B., Myczkowski, M. L., Nitsche, M. A., Pascual-Leone, A., \& et al. (2008). A randomized, double-blind clinical trial on the efficacy of cortical direct current stimulation for the treatment of major depression. International Journal of Neuropsychopharmacology, 11(2), 249.

Brunoni, A. R., Amadera, J., Berbel, B., Volz, M. S., Rizzerio, B. G., \& Fregni, F. (2011). A systematic review on reporting and assessment of adverse effects associated with transcranial direct current stimulation. International Journal of Neuropsychopharmacology, 14(8), 1133-1145.

Datta, A., Bansal, V., Diaz, J., Patel, J., Reato, D., \& Bikson, M. (2009). Gyri-precise head model of transcranial direct current stimulation: improved spatial focality using a ring electrode versus conventional rectangular pad. Brain Stimulation, 2(4), 201-207.

Del Casale, A., Kotzalidis, G. D., Rapinesi, C., Serata, D., Ambrosi, E., Simonetti, A., \& et al. (2011). Functional neuroimaging in obsessive-compulsive disorder. Neuropsychobiology, 64(2), 61-85.

Dobson, K. E., Mohammadkhani, P. (2007). Psychometrics characteristics of BDI-II. Journal of Rehabilitation, 8 (29), 82-8.

Foa, E. B., Liebowitz, M. R., \& Kozak, M. J. (2005). Randomized, placebo-controlled trial of exposure and ritual prevention, clomipramine, and their combination in the treatment of obsessivecompulsive disorder. Amercian Journal of Psychiatry, 162(1), 151161

Fontenelle, I., Fontenelle, L., \& Borges, M. (2010). Quality of life and symptom dimensions of patients with obsessive-compulsive disorder. Psychiatry Research, 179(2), 198-203.

Fregni, F., Boggio, P. S., Nitsche, M. A., Marcolin, M. A., Rigonatti, S. P., \& Pascual-Leone, A. (2006). Treatment of major depression with transcranial direct current stimulation. Bipolar Disorders, $8(2), 203-204$.

Goodman, W. K., Price, L. H., Rasmussen, S. A., Mazure, C., Delgado, P., Heninger, G. R., et al. (1989). The yale-brown obsessive compulsive scale: II. Validity. Archives of General Psychiatry, 46(11), 1012-1016.

Hamilton, M. A. X. (1959). The assessment of anxiety states by rating. British Journal of Medical Psychology, 32(1), 50-55.

Ivarsson, T., Melin, K., \& Wallin, L. (2008). Categorical and dimensional aspects of co-morbidity in obsessive-compulsive disorder (OCD). European Child \& Adolescent Psychiatry, 17(1), 20-31.

Jaafari, N., Rachid, F., Rotge, J. Y., Polosan, M., El-Hage, W., Belin, D., et al. (2012). Safety and efficacy of repetitive transcranial magnetic stimulation in the treatment of obsessive-compulsive disorder: A review. World Journal of Biological Psychiatry, 13(3), 164-177.

Kavyani, H., Mossavi, A. S., \& Mohit, A. (2001). [Psychological Scales and Interviews (Persian)]. Tehran: Sana Publications.

Kuo, M. F., Paulus, W., \& Nitsche, M. A. (2014). Therapeutic effects of non-invasive brain stimulation with direct currents (tDCS) in neuropsychiatric diseases. Neuroimage, 85, 948-960.

Langley, A. K., Lewin, A. B., Bergman, R. L., Lee, J. C., \& Piacentini, J. (2010). Correlates of comorbid anxiety and externalizing disorders in childhood obsessive compulsive disorder. European Child \& Adolescent Psychiatry, 19(8), 637-645.

Loo, C. K., Alonzo, A., Martin, D., Mitchell, P. B., Galvez, V., \& Sachdev, P. (2012). Transcranial direct current stimulation for depres- 
sion: 3-week, randomised, sham-controlled trial. British Journal of Psychiatry, 200(1), 52-59.

Mathers, C. D., Bernard, C., Iburg, K. M., Inoue, M., Ma Fat, D., Shibuya, K., \& et al. (2003). Global burden of disease in 2002: data sources, methods and results. Geneva: World Health Organization.

Milad, M. R., \& Rauch, S. L. (2012). Obsessive-compulsive disorder: beyond segregated cortico-striatal pathways. Trends in Cognitive Sciences, 16(1), 43-51.

Mondino, M., Haesebaert, F., Poulet, E., Saoud, M., \& Brunelin, J. (2015). Efficacy of cathodal transcranial direct current stimulation over the left orbitofrontal cortex in a patient with treatmentresistant obsessive-compulsive disorder. Journal of ECT, 31(4), 271-272.

Nitsche, M. A., Nitsche, M., Klein, C., Tergau, F., Rothwell, J., \& Paulus, W. (2003). Level of action of cathodal DC polarization induced inhibition of the human motor cortex. Clinical Neurophysiology, 114(4), 600-604.

Nitsche, M. A., \& Paulus, W. (2000). Excitability changes induced in the human motor cortex by weak transcranial direct current stimulation. Journal of Physiology, 527(3), 633-639.

Peris, T. S., Bergman, R. L., Asarnow, J. R., Langley, A., McCracken, J. T., \& Piacentini, J. (2010). Clinical and cognitive correlates of depressive symptoms among youth with obsessive compulsive disorder. Journal of Clinical Child \& Adolescent Psychology, 39(5), 616-626.

Polanía, R., Paulus, W., \& Nitsche, M. A. (2012). Modulating cortico-striatal and thalamo-cortical functional connectivity with transcranial direct current stimulation. Human Brain Mapping, 33(10), 2499-2508.

Priori, A., Hallet, M., \& Rothwell, J. C. (2009). Repetitive transcranial magnetic stimulation or trancranial direct current stimulation? Brain Stimulation, 2(4), 241-245.

Rajezi Esfahani, S., Motaghipour, Y., Kamkari, K., Zahiredin, A., \& Janbozorgi, M. (2012). Reliability and validity of the Persian version of the yale-Brown Obsessive-Compulsive Scale (Y-BOCS). Iranian Journal of Psychiatry and Clinical Psychology, 17(4), 297-303.

Ruscio, A., Sten, N. N., ldvin, D., Chiu, W., \& Kessler, R. (2010). The epidemiology of obsessive-compulsive disorder in the National Comorbidity Survey Replication. Molecular Psychiatry, 15(1), 5363.

Sakai, Y., Narumoto, J., Nishida, S., Nakamae, T., Yamada, K., Nishimura, $\mathrm{T}$., et al. (2011). Corticostriatal functional connectivity in non-medicated patients with obsessive-compulsive disorder. European Psychiatry, 26(7), 463-469.

Senco, N. M., Huang Y., D’Urso, G., Parra, L. C., Bikson, M., Mantovani, A., et al. (2015). Transcranial direct current stimulation in obsessive-compulsive disorder: emerging clinical evidence and considerations for optimal montage of electrodes. Expert Review of Medical Devices, 1-11.

Shah, D. B., Pesiridou, A., \& Baltuch, G. H. (2008). Functional neurosurgery in the treatment of severe obsessive-compulsive disorder and major depression. Psychiatry, 5(9), 24-33.

Storch, E. A., Merlo, L. J., Larson, M. J., Geffken, G. R., Lehmkuhl, H. D., Jacob, M. L., et al. (2008). Impact of comorbidity on cognitivebehavioral therapy response in pediatric obsessive-compulsive disorder. Journal of the American Academy of Child \& Adolescent Psychiatry, 47(5), 583-592.
Swedo, S. E., Pietrini, P., Leonard, H. L., Schapiro, M. B., Rettew, D. C., \& Goldberger, E. L. (1992). Cerebral glucose metabolism in childhoodonset obsessive-compulsive disorder. Revisualization during pharmacotherapy. Archieve of Genetic Psychiatry, 49(9), 690-694.

Volpato, C., Piccione, F., Cavinato, M., Duzzi, D., Schiff, S., Foscolo, L., et al. (2013). Modulation of affective symptoms and resting state activity by brain stimulation in a treatment-resistant case of obsessive-compulsive disorder. Neurocase, 19(4), 360-370. 